\title{
Interdependence in a Japanese cultural context: Distinguishing between preferences and expectations
}

\author{
Hirofumi HASHIMOTO* \\ Yasuda Women's University
}

\begin{abstract}
The purpose of the current study was to clarify whether interdependent behavior among the Japanese is aligned with their expectations regarding others' behavior or their own preferences. The participants were privately asked about their preference for independence or interdependence and their expectations regarding others' independent or interdependent behavior. Then, they were asked to publicly express whether their own behavior was indicative of independence or interdependence. When comparing the participants' preferences, expectations, and actual behavior, I found that interdependence was only evident in their expectations and public behavior; i.e., the participants answered that they preferred independence rather than interdependence, whereas they expected that others are interdependent and identified themselves as interdependent in public. These findings suggest that interdependent behavior among the Japanese is based on their expectations regarding others' behavior rather than their own preferences.
\end{abstract}

Key Words: interdependence, preference, expectation

\section{Introduction}

Over the last quarter century, cultural psychologists have found a broad range of cultural differences in cognitions and behavior reflecting a cultural preference for or values related to independence and interdependence (Kim \& Markus, 1999; Markus \& Kitayama, 1991; also see Cross, Hardin, \& Gercek-Swing, 2011 for a review). Through their research, they have demonstrated how cultural mandates (i.e., ideals or general goals) for independence (e.g., among North Americans) or interdependence (e.g., among East Asians) influence people's perceptions of reality and their motivations to behave in particular ways (e.g., Kitayama, Park, Sevincer, Karasawa, \& Uskul, 2009; Kitayama, Karasawa, Curhan, Ryff, \& Markus, 2010). Their findings have helped us understand how people's cognitions and behaviors differ among cultures; however, the explanations for why these cultural differences are observed are contentious. In the current study, I propose one possible explanation — that is, from an adaptationist perspective (Hashimoto \& Yamagishi, 2013, 2016; Yamagishi, 2011; Yamagishi \& Hashimoto, 2016; Yamagishi, Hashimoto, \& Schug, 2008; Yamagishi et al., 2012), I assume that culture-specific behavior commonly considered as "interdependent" (or "not independent") in cultural psychology has adaptive value. This means that interdependent behavior acts as part of an adaptive strategy to avoid creating a negative impression or reputation among members of one's community. Accordingly, I hypothesize that interdependent behavior in the Japanese cultural context is aligned with people's expectations regarding others' behavior, rather than their own personal preferences for behaving that way.

* Corresponding Author e-mail: hashimoto-h@yasuda-u.ac.jp 


\section{Cultural Agents and Cultural Game Players}

A well-known assumption in cultural psychology is that there is consistency between individual psychological tendencies (e.g., individuals' preferences, values, or motivations) and culturally shared meaning systems (see also Kitayama, Markus, Matsumoto, \& Norasakkunkit, 1997; Uchida, Kitayama, Mesquita, Reyes, \& Morling, 2008). According to this cycle of "mutually constituted culture and psychology," which is one of the core theories of cultural psychology, the individual level often cannot be separated from the cultural level (Markus \& Kitayama, 1998). This view, which Yamagishi and colleagues termed the "cultural agent view” (see Hashimoto, Li, \& Yamagishi, 2011; Yamagishi, 2011), assumes the existence of a cultural agent with culturally specific preferences and focuses on how the consistency between culture-wide traits and individuals' own psychology develops through socialization.

Yamagishi and colleagues (Hashimoto et al., 2011; Hashimoto \& Yamagishi, 2015; Yamagishi et al., 2008, 2012) proposed an alternative approach connecting culturally shared beliefs with behaviors that argues that humans are cultural game players who pursue their goals in anticipation of others' responses. According to this so-called "cultural game player view" (see Hashimoto et al., 2011; Yamagishi, 2011), culturally specific behaviors are adaptive strategies rather than simple expressions of cultural preferences, and behaviors are tailored to enable players to succeed in socially adaptive tasks and thereby acquire valuable resources from others.

Based on this view, Hashimoto (2011) showed that Japanese people revealed their preference for independence over interdependence, while they expected that others would behave interdependently. In this study, participants were asked to complete independent and interdependent selfconstrual scales in three contexts. First, the participants answered about their actual selves. Second, the same participants answered the same scales from the viewpoint of the ideal self - the person they wanted to be. Finally, they answered the same scales from the viewpoint of general other people. Through comparisons of participants' responses in these three contexts, Hashimoto (2011) found that Japanese respondents believed that the majority of other people are more interdependent than independent. This pattern was found to be completely reversed in their ideal-self responses. In other words, the kind of person the participants wanted to be was found to be one who endorses the traits of independence more strongly than independence. Finally, their actual self was found to fall between the two extremesthe ideal self and others' expected responses. Their actual self was more interdependent than independent, but the difference between the two was not as pronounced as in the case of others' expected responses. Based on these findings, Hashimoto and Yamagishi (2015) examined Japanese and American individuals' preferences and expectations regarding independence and interdependence. They found that Japanese and American participants both preferred independence to interdependence; that is, no cultural difference was found. On the other hand, a strong cultural difference was found in their expectations about how others would judge independent and interdependent people. Specifically, a "preference-expectation reversal" emerged among the Japanese university student participants: while they preferred independence to interdependence, they expected that others would evaluate interdependence more positively than independence. Such a reversal was not observed among the American participants, who preferred independence and expected others to evaluate independence more positively. These findings suggested that even Japanese participants who personally prefer independence do act interdependently in order to avoid the expected negative responses from others.

\section{The Current Study}

The purpose of the current study was to conceptually replicate the previously found preference-expectation reversal among Japanese individuals, and further expand the findings by experimentally demonstrating that Japanese people behave interdependently in public even if they prefer independence. Previous studies argued that expectations regarding others' behavior play an important role in interdependent behavior among Japanese people; however, the question immediately arises: why do those who prefer independence expect the majority of other people to be more interdependent or expect negative responses from others, many of whom may personally prefer independence than interdependence? A possible, indirect answer is that those who personally prefer independence do actually behave interdependently in public. People's expectations regarding others' behavior basically stem from others' observable behavior. The studies by Hashimoto (2011) and Hashimoto and 
Yamagishi (2015) did not examine how publicly displayed Japanese behavior fits into the preference-expectation reversal. According to the cultural game player view, in the current study, it was predicted that Japanese people's observable behavior would be characterized as interdependent, and that this would be aligned with their expectations of others' behavior, regardless of their own preferences. Specifically, the aim of this study was to test the following two hypotheses.

Hypothesis 1. There is a clear preference-expectation reversal among the Japanese - that is, Japanese people prefer independence but expect others to behave interdependently.

Hypothesis 2. Japanese people's observable behavior would be characterized as interdependent.

\section{Method}

One hundred and fifty-seven Japanese university students (72 women, 75 men, and 10 of unspecified sex; mean age $=19.4$ years) participated in this study. This study was conducted as part of a supplementary lecture on social psychology; students attending the lectures could freely chose the time slot. Six experimental sessions $(N=12$ to 53)1) were conducted in total. After the brief lecture, participants were told that their anonymity would be ensured, that their decision to participate is voluntary, and that they could freely stop participating at any point. All the students who attended the lecture agreed to participate; at that point, the experimenter distributed the questionnaire to them.

The questionnaire contained descriptions of independent and interdependent persons as Person A and Person B, respectively, which were derived from the study by Hashimoto and Yamagishi (2015), along with the related items. After the participants read the descriptions, they were asked to privately indicate their preference for independence and interdependence as well as their expectations regarding others' behaviors for the same. They answered these questions categorically (i.e., by choosing either Person A or Person B). More specifically, they were asked if, given a chance, they would want to act more like Person A or Person B (i.e., preference), and whether most people's actions are closer to those of Person A or Person B (i.e., expected behavior). Upon completing the questionnaire, the participants placed it in an envelope, which they sealed to ensure their anonymity.

At this point, the participants were asked to answer a final question regarding their own behavior: the experimenter showed the descriptions of Person A and Person B on a PowerPoint slide in order that all the participants could see them. They were then asked to publicly express whether their own behavior could be classified as independent or interdependent, based on those definitions. To do this, the experimenter publicly asked those who believed that their actual behavior is more reflective of an independent person (i.e., Person A) to stand up and bring their envelope to the desk in the front; the experimenter asked the same of those who believed their behavior to be more interdependent (i.e., indicative of Person B). ${ }^{2)}$

\section{Results}

As shown in Figure 1, I confirmed the preference-expectation reversal found in past research. The participants preferred independent (i.e., chose Person A; 66.2\%) rather than interdependent (i.e., chose Person B; 33.8\%) behavior, and the difference from $50 \%$ was significant, $\chi^{2}(1)=16.57, p<.001$. Regardless of their preference, the participants expected others to behave interdependently $(69.4 \%)$ rather than independently (30.6\%); the difference from $50 \%$ for this comparison was also significant, $\chi^{2}(1)=23.70, p<.001$. These results supported Hypothesis 1 .

The results clearly indicated that the participants also identified themselves publicly as interdependent (77.7\%) rather than independent (22.3\%); the difference from $50 \%$ was significant, $\chi^{2}(1)=48.21, p<.001$. This result supported Hypothesis 2 .

\section{Discussion}

Based on the cultural game player view, the current study attempted to replicate the preference-expectation reversal among Japanese participants (Hypothesis 1) and determine whether Japanese people do actually behave interdependently in public (Hypothesis 2). The results clearly supported both hypotheses, thereby confirming the preferenceexpectation reversal and further finding that the participants

1) There were no significant differences between sessions among the dependent variables.

2) The order in which the participants were asked to answer was counterbalanced; it did not affect their behavior. 


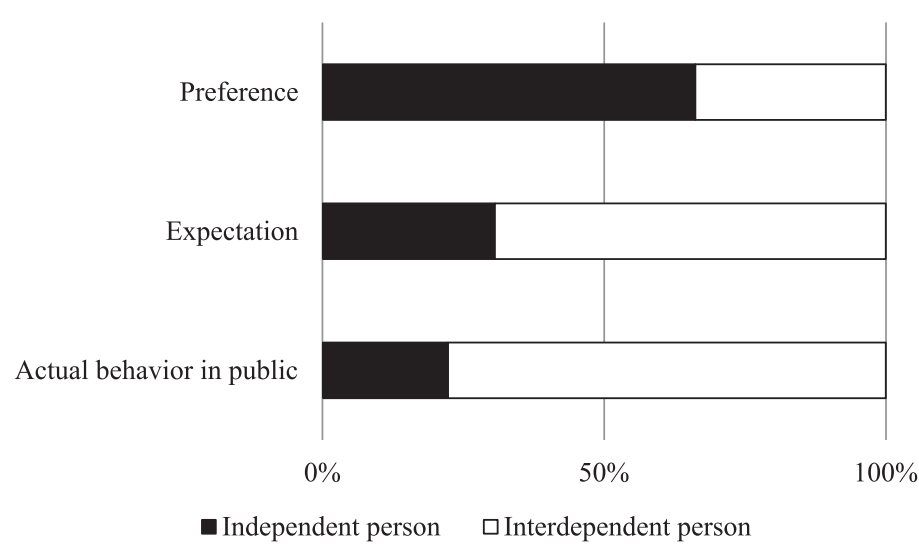

Figure 1 Percentage of participants who chose the independent or interdependent person $(n=157)$

express interdependent behavior in public. Past findings on the preference-expectation reversal, as discussed in the Introduction section, suggest that Japanese people tend to behave interdependently in an attempt to avoid negative responses. Taking this finding together with the results of the current study, it is reasonable to interpret that Japanese people behave interdependently in public because they anticipate others' responses and adjust their behavior according to the responses they expect from others. According to Yamagishi's social niche construction theory (Yamagishi, 2011; Yamagishi \& Hashimoto, 2016), East Asian societies are characterized as closed collectivistic societies (i.e., consisting of groups and relationships that are closed to outsiders). In such a society, security within particular groups and relationships is what provides individuals with their required resources, and there are few to no alternative external sources that individuals can access for resources (Greif, 1994; Yamagishi \& Yamagishi, 1994; Yamagishi, Cook, \& Watabe, 1998). Accordingly, being excluded from one's group or relationships is incredibly costly, as excluded individuals have nowhere else to turn to for their required resources. Thus, to minimize the risk of being excluded, individuals must be sensitive to others' attitudes toward them and avoid offending others. The findings of the current study are consistent with these arguments.

Certain limitations of the current study should be noted. First, the findings in the current study are limited to the Japanese context; therefore, further research is needed to examine whether these results can be replicated in other East Asian samples. Second, "interdependence" in the
Japanese cultural context should be more narrowly defined and examined. Hashimoto and Yamagishi (2013, 2016) identified two distinct aspects of interdependence: harmony seeking and rejection avoidance. Future research should examine which of these aspects of interdependence are identified in the "preference-expectation reversal." Third, the current study does not have a definite answer for why Japanese people prefer independence rather than interdependence in the first place. Whether the questionnaire used in the current study adequately measured the participants' preference is arguable. Future research should determine the answers to these questions.

Finally, while in the current study, it was found that Japanese individuals' behavior in public aligned with their expectations regarding others' behavior more than they did with their preferences, I do not mean to suggest that cultural preferences are in any way meaningless. Indeed, past studies in cultural psychology have clearly illustrated their importance. In this sense, when and in what ways cultural preferences or beliefs are also particularly important is an interesting and potentially fruitful future research question.

\section{Acknowledgment}

This study was supported by Grants-in-Aid 17K13909 from the Japan Society for the Promotion of Science.

\section{References}

Cross, S. E., Hardin, E. E., \& Gercek-Swing, B. (2011). The what, how, why, and where of self-construal. Personality and Social Psychology Review, 15, 142-179. 
Greif, A. (1994). Cultural beliefs and the organization of society: A historical and theoretical reflection on collectivist and individualist societies. Journal of Political Economy, 102, 912-950.

Hashimoto, H. (2011). Interdependence as a self-sustaining set of beliefs. Japanese Journal of Experimental Social Psychology, 50, 182-193 (in Japanese).

Hashimoto, H., Li, Y., \& Yamagishi, T. (2011). Beliefs and preferences in cultural agents and cultural game players. Asian Journal of Social Psychology, 14, 140-147.

Hashimoto, H., \& Yamagishi, T. (2013). Two faces of interdependence: Harmony seeking and rejection avoidance. Asian Journal of Social Psychology, 16, 142-151.

Hashimoto, H., \& Yamagishi, T. (2015). Preferenceexpectation reversal in the ratings of independent and interdependent individuals: A USA-Japan comparison. Asian Journal of Social Psychology, 18, 115-123.

Hashimoto, H., \& Yamagishi, T. (2016). Duality of independence and interdependence: An adaptationist perspective. Asian Journal of Social Psychology, 19, 286297.

Kim, H., \& Markus, H. R. (1999). Deviance or uniqueness, harmony or conformity? A cultural analysis. Journal of Personality and Social Psychology, 77, 785-800.

Kitayama, S., Karasawa, M., Curhan, K. B., Ryff, C. D., \& Markus, H. R. (2010). Independence and interdependence predict health and wellbeing: Divergent patterns in the United States and Japan. Frontiers in Psychology, 1: 163. doi: 10.3389/fpsyg.2010.00163

Kitayama, S., Markus, H. R., Matsumoto, H., \& Norasakkunkit, V. (1997). Individual and collective processes in the construction of the self: Self-enhancement in the United States and self-criticism in Japan. Journal of Personality and Social Psychology, 72, 1245-1267.

Kitayama, S., Park, H., Sevincer, A. T., Karasawa, M., \& Uskul, A. K. (2009). A cultural task analysis of implicit independence: Comparing North America, Western Europe, and East Asia. Journal of Personality and Social Psychology, 97, 236-255.

Markus, H. R., \& Kitayama, S. (1991). Culture and the self: Implications for cognition, emotion, and motivation. Psychological Review, 98, 224-253.

Markus, H. R., \& Kitayama, S. (1998). The cultural psychology of personality. Journal of Cross-Cultural Psychology, 29, 63-87.

Uchida, Y., Kitayama, S., Mesquita, B., Reyes, J. A. S., \& Morling, B. (2008). Is perceived emotional support beneficial? Well-being and health in independent and interdependent cultures. Personality and Social Psychology Bulletin, 34, 741-754.

Yamagishi, T. (2011). Micro-macro dynamics of the cultural construction of reality: A niche construction approach to culture. Advances in Culture and Psychology, 1, 251308.

Yamagishi, T., Cook, K. S., \& Watabe, M. (1998). Uncertainty, trust and commitment formation in the United States and Japan. American Journal of Sociology, 104, 165-194.

Yamagishi, T., Hashimoto, H. (2016). Social niche construction. Current Opinion in Psychology, 8, 119-124.

Yamagishi, T., Hashimoto, H., Schug, J. (2008). Preference vs. strategies as explanations for culture-specific behavior. Psychological Science, 19, 579-584.

Yamagishi, T., Hashimoto, H., Cook, K. S., Kiyonari, T., Shinada, M., Mifune, N., Inukai, K., Takagishi, H., Horita, Y., \& Li, Y. (2012). Modesty in self-presentation: A comparison between the USA and Japan. Asian Journal of Social Psychology, 15, 60-68.

Yamagishi, T., \& Yamagishi, M. (1994). Trust and commitment in the United States and Japan. Motivation and Emotion, 18, 129-166. 